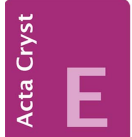

\title{
Redetermination of the crystal structure of $\beta$-zinc molybdate from single-crystal $X$-ray diffraction data
}

\section{Olfa Mtioui-Sghaier, ${ }^{\mathrm{a}}$ Rafael Mendoza-Meroño, ${ }^{\mathrm{a}}$ Lilia Ktari, ${ }^{\text {b }}$ Mohamed Dammak ${ }^{\text {b }}$ and Santiago García- Granda $^{\mathrm{a} *}$}

aDepartamento de Química Física y Analítica, Facultad de Química, Universidad de Oviedo-CINN, C/ Julián Clavería, 8, 33006 Oviedo, Spain, and ${ }^{\mathbf{b}}$ Laboratoire de Chimie Inorganique, Faculté des Sciences de Sfax, Route de Soukra, 3000 Sfax, Tunisia. *Correspondence e-mail: sgg@uniovi.es

Received 8 May 2015; accepted 21 June 2015

Edited by M. Weil, Vienna University of Technology, Austria

The crystal structure of the $\beta$-polymorph of $\mathrm{ZnMoO}_{4}$ was redetermined on the basis of single-crystal X-ray diffraction data. In comparison with previous powder X-ray diffraction studies [Katikaneani \& Arunachalam (2005). Eur. J. Inorg. Chem. pp. 3080-3087; Cavalcante et al. (2013). Polyhedron, 54, 13-25], all atoms were refined with anisotropic displacement parameters, leading to a higher precision with respect to bond lengths and angles. $\beta$ - $\mathrm{ZnMoO}_{4}$ adopts the wolframite structure type and is composed of distorted $\mathrm{ZnO}_{6}$ and $\mathrm{MoO}_{6}$ octahedra, both with point group symmetry 2 . The distortion of the octahedra is reflected by variation of bond lengths and angles from 2.002 (3)-2.274 (4) $\AA$, 80.63 (11)-108.8 (2) ${ }^{\circ}$ for equatorial and $158.4(2)-162.81(14)^{\circ}$ for axial angles $\left(\mathrm{ZnO}_{6}\right)$, and of 1.769 (3)-2.171 (3) ^, 73.39 (16)-104.7 (2), 150.8 (2)$164.89(15)^{\circ}\left(\mathrm{MoO}_{6}\right)$, respectively. In the crystal structure, the same type of $\mathrm{MO}_{6}$ octahedra share edges to built up zigzag chains extending parallel to [001]. The two types of chains are condensed by common vertices into a framework structure. The crystal structure can alternatively be described as derived from a distorted hexagonally closed packed arrangement of the $\mathrm{O}$ atoms, with $\mathrm{Zn}$ and Mo in half of the octahedral voids.

Keywords: crystal structure; redetermination; $\beta-\mathrm{ZnMoO}_{4}$; hydrothermal synthesis; wolframite structure type.

CCDC reference: 1408028

\section{Related literature}

Most molybdates of divalent cations crystallize either in the scheelite-type or in the wolframite-type (Macavei \& Schulz,
1993). Zinc molybdate $\left(\mathrm{ZnMoO}_{4}\right)$ is an inorganic semiconductor. It adopts the wolframite-type of structure (Keeling, 1957) and is dimorphic. The two phases, referred to as $\alpha$ (triclinc symmetry) and $\beta$ - (monoclinic symmetry), can be selectively obtained by controlling the synthetic conditions (Abrahams et al., 1967; Zhang et al., 2010). Previous crystal structure refinements of $\beta-\mathrm{ZnMoO}_{4}$, based on X-ray powder diffraction data, were reported by Cavalcante et al. (2013) and Katikaneani \& Arunachalam (2005). For structure refinement of $\mathrm{ZnWO}_{4}$, isotypic with the title compound, see: Trots et al. (2009).

\section{Experimental}

2.1. Crystal data

$\mathrm{ZnMoO}_{4}$

$M_{r}=225.31$

Monoclinic, $P 2 / c$

$a=4.6980(3) \AA$

$b=5.7380(4) \AA$

$c=4.8960(4) \AA$

$\beta=90.311(7)^{\circ}$

$$
\begin{aligned}
& V=131.98(2) \AA^{3} \\
& Z=2 \\
& \text { Mo } K \alpha \text { radiation } \\
& \mu=13.62 \mathrm{~mm}^{-1} \\
& T=293 \mathrm{~K} \\
& 0.08 \times 0.06 \times 0.03 \mathrm{~mm}
\end{aligned}
$$

\subsection{Data collection}

Oxford Diffraction Xcalibur CCD diffractometer

Absorption correction: multi-scan (CrysAlis PRO; Oxford Diffraction, 2014)

$T_{\min }=0.905, T_{\max }=1.000$

\subsection{Refinement}

$R\left[F^{2}>2 \sigma\left(F^{2}\right)\right]=0.028$

$w R\left(F^{2}\right)=0.068$

$S=1.10$

405 reflections

1207 measured reflections 405 independent reflections 358 reflections with $I>2 \sigma(I)$ $R_{\text {int }}=0.036$

Data collection: CrysAlis CCD (Oxford Diffraction, 2014); cell refinement: CrysAlis RED (Oxford Diffraction, 2014); data reduction: CrysAlis RED; program(s) used to solve structure: SIR2011 (Burla et al., 2012); program(s) used to refine structure: SHELXL2014 (Sheldrick, 2015); molecular graphics: DIAMOND (Brandenburg \& Putz, 1999); software used to prepare material for publication: WinGX (Farrugia, 2012), publCIF (Westrip, 2010) and PARST (Nardelli, 1995).

\section{Acknowledgements}

We acknowledge financial support from the Spanish Ministerio de Economía y Competitividad (MAT2013-40950-R), Gobierno del Principado de Asturias (GRUPIN14-060) and ERDF.

Supporting information for this paper is available from the IUCr electronic archives (Reference: WM5159). 


\section{References}

Abrahams, S. C. (1967). J. Chem. Phys. 46, 2052-2063.

Brandenburg, K. \& Putz, H. (1999). DIAMOND. Crystal Impact GbR, Bonn, Allemagne.

Burla, M. C., Caliandro, R., Camalli, M., Carrozzini, B., Cascarano, G. L., Giacovazzo, C., Mallamo, M., Mazzone, A., Polidori, G. \& Spagna, R. (2012). J. Appl. Cryst. 45, 357-361.

Cavalcante, L. S., Moraes, E., Almeida, M. A. P., Dalmaschio, C. J., Batista, N. C., Varela, J. A., Longo, E., Siu Li, M., Andrés, J. \& Beltrán, A. (2013). Polyhedron, 54, 13-25.

Farrugia, L. J. (2012). J. Appl. Cryst. 45, 849-854.

Katikaneani, P. \& Arunachalam, R. (2005). Eur. J. Inorg. Chem. pp. 30803087.
Keeling, R. O. (1957). Acta Cryst. 10, 209-213.

Macavei, J. \& Schulz, H. (1993). Z. Kristallogr. 207, 193-208.

Nardelli, M. (1995). J. Appl. Cryst. 28, 659.

Oxford Diffraction (2014). CrysAlis PRO, CrysAlis CCD and CrysAlis RED. Oxford Diffraction Ltd., Abingdon, England.

Sheldrick, G. M. (2015). Acta Cryst. C71, 3-8.

Trots, D. M., Senyshyn, A., Vasylechko, L., Niewa, R., Vad, T., Mikhailik, V. B. \& Kraus, H. (2009). J. Phys. Condens. Matter, 21, 325402.

Westrip, S. P. (2010). J. Appl. Cryst. 43, 920-925.

Zhang, G., Yu, S., Yang, Y., Jiang, W., Zhang, S. \& Huang, B. (2010). J. Cryst. Growth, 312, 1866-1874. 


\section{supporting information}

Acta Cryst. (2015). E71, i6-i7 [doi:10.1107/S205698901501186X]

\section{Redetermination of the crystal structure of $\beta$-zinc molybdate from single-crystal X-ray diffraction data}

\section{Olfa Mtioui-Sghaier, Rafael Mendoza-Meroño, Lilia Ktari, Mohamed Dammak and Santiago} García-Granda

\section{S1. Synthesis and crystallization}

Reagents were used as commercial sources with no further purification. An aqueous solution was prepared by a mixture

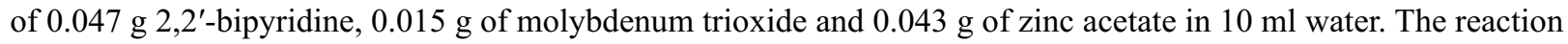
mixture was stirred at room temperature to homogeneity, then transferred into a teflon-lined stainless steel vessel (40 ml) and heated to $453 \mathrm{~K}$ for $48 \mathrm{~h}$ under autogenous pressure and after-wards cooled slowly to room temperature. The resulting material was obtained as colorless single-crystals without side products. The solid was filtered off, washed thoroughly with distilled water, and finally air-dried at room temperature.

\section{S2. Refinement}

The remaining maximum and minimum electron densities were found $0.77 \AA$ and $0.90 \AA$, respectively, from the $\mathrm{O} 1$ atom. $\mathrm{O} 1$ is located on the center of a tree-metal triangle, bridging one Mo and two $\mathrm{Zn}$ atoms. 


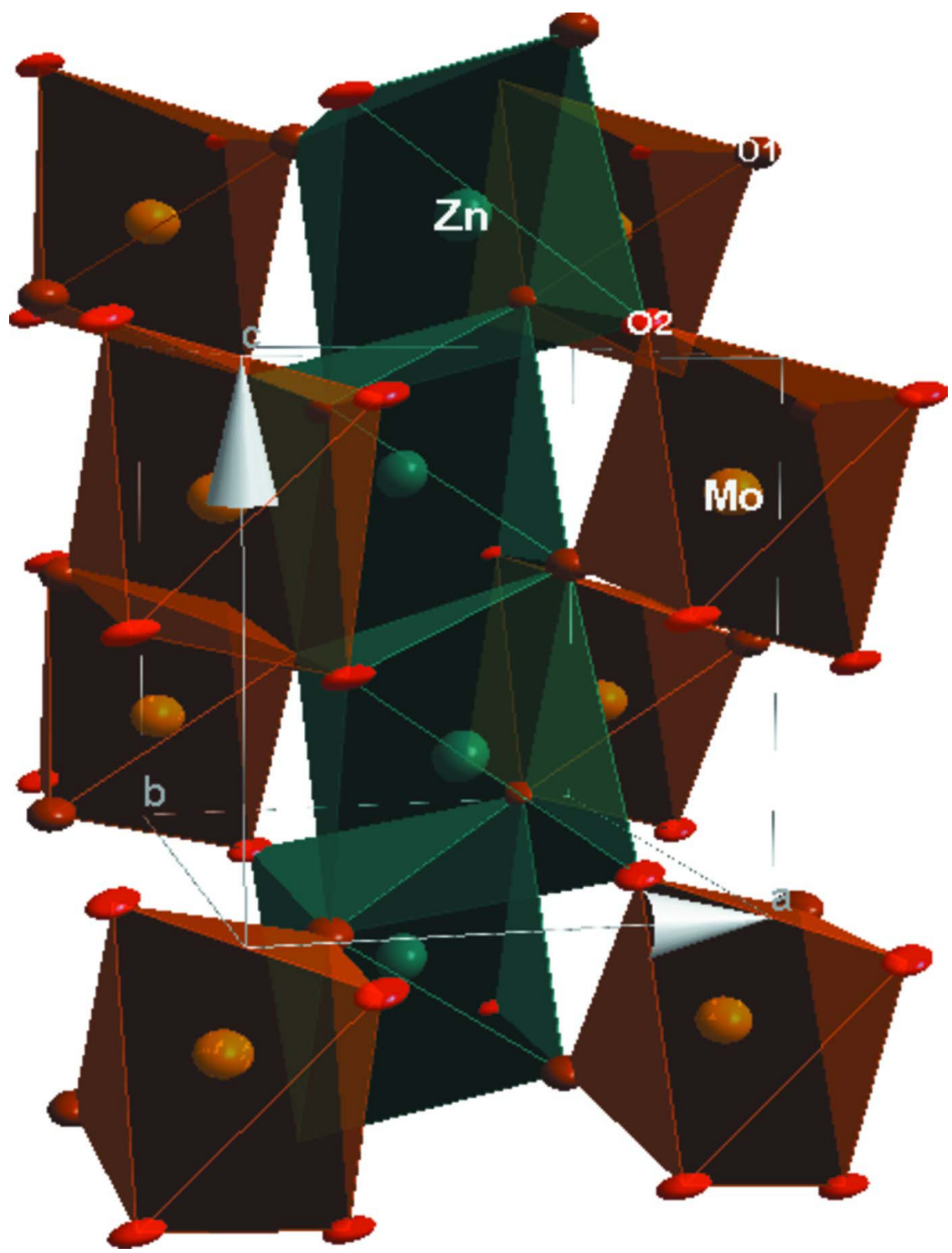

\section{Figure 1}

A view of the crystal structure of $\beta-\mathrm{ZnMoO}_{4}$. Anisotropic displacement parameters are drawn at the $50 \%$ probability level.

\section{$\beta$-Zinc molybdate}

\section{Crystal data}

$\mathrm{MoO}_{4} \mathrm{Zn}$

$M_{r}=225.31$

Monoclinic, $P 2 / c$

$a=4.6980(3) \AA$

$b=5.7380(4) \AA$

$c=4.8960(4) \AA$

$\beta=90.311(7)^{\circ}$

$V=131.98(2) \AA^{3}$

$Z=2$
$F(000)=208$

$D_{\mathrm{x}}=5.670 \mathrm{Mg} \mathrm{m}^{-3}$

Mo $K \alpha$ radiation, $\lambda=0.71073 \AA$

Cell parameters from 570 reflections

$\theta=3.6-31.1^{\circ}$

$\mu=13.62 \mathrm{~mm}^{-1}$

$T=293 \mathrm{~K}$

Prism, colourless

$0.08 \times 0.06 \times 0.03 \mathrm{~mm}$ 


\section{Data collection}

Oxford Diffraction Xcalibur CCD diffractometer

Radiation source: Enhance (Mo) X-ray Source Detector resolution: 10.2673 pixels $\mathrm{mm}^{-1}$

$\omega-$ and $\varphi$-scans

Absorption correction: multi-scan

(CrysAlis PRO; Oxford Diffraction, 2014)

$T_{\min }=0.905, T_{\max }=1.000$

\section{Refinement}

Refinement on $F^{2}$

Least-squares matrix: full

$R\left[F^{2}>2 \sigma\left(F^{2}\right)\right]=0.028$

$w R\left(F^{2}\right)=0.068$

$S=1.10$

405 reflections

29 parameters

Special details

Geometry. All e.s.d.'s (except the e.s.d. in the dihedral angle between two 1.s. planes) are estimated using the full covariance matrix. The cell e.s.d.'s are taken into account individually in the estimation of e.s.d.'s in distances, angles and torsion angles; correlations between e.s.d.'s in cell parameters are only used when they are defined by crystal symmetry. An approximate (isotropic) treatment of cell e.s.d.'s is used for estimating e.s.d.'s involving l.s. planes.

Fractional atomic coordinates and isotropic or equivalent isotropic displacement parameters $\left(\AA^{2}\right)$

\begin{tabular}{lllll}
\hline & $x$ & $y$ & $z$ & $U_{\text {iso }} * / U_{\text {eq }}$ \\
\hline Mo1 & 1.0000 & $0.81190(10)$ & 0.2500 & $0.00507(18)$ \\
Zn1 & 1.5000 & $0.69182(15)$ & 0.7500 & $0.0092(2)$ \\
O1 & $1.2538(7)$ & $0.6236(6)$ & $0.4014(7)$ & $0.0080(7)$ \\
O2 & $0.7835(7)$ & $0.8950(6)$ & $0.5603(7)$ & $0.0058(7)$ \\
\hline
\end{tabular}

Atomic displacement parameters $\left(\AA^{2}\right)$

\begin{tabular}{lllllll}
\hline & $U^{11}$ & $U^{22}$ & $U^{33}$ & $U^{12}$ & $U^{13}$ & $U^{23}$ \\
\hline Mo1 & $0.0065(3)$ & $0.0045(3)$ & $0.0041(3)$ & 0.000 & $-0.0002(2)$ & 0.000 \\
Zn1 & $0.0087(4)$ & $0.0116(4)$ & $0.0073(4)$ & 0.000 & $0.0009(3)$ & 0.000 \\
O1 & $0.0089(17)$ & $0.0110(16)$ & $0.0041(17)$ & $0.0006(14)$ & $0.0003(13)$ & $-0.0004(14)$ \\
O2 & $0.0088(16)$ & $0.0064(14)$ & $0.0021(16)$ & $-0.0005(13)$ & $0.0016(12)$ & $-0.0010(13)$ \\
\hline
\end{tabular}

Geometric parameters $\left(\AA,{ }^{\circ}\right)$

\begin{tabular}{|c|c|c|c|}
\hline Mo1-O1 ${ }^{\mathrm{i}}$ & $1.769(3)$ & $\mathrm{Zn} 1-\mathrm{O} 2^{\mathrm{iv}}$ & $2.002(3)$ \\
\hline Mo1-O1 & $1.769(3)$ & $\mathrm{Zn} 1-\mathrm{O} 2^{\mathrm{v}}$ & $2.002(3)$ \\
\hline Mo1-O2 & $1.894(3)$ & $\mathrm{Zn} 1-\mathrm{O} 1^{\mathrm{vi}}$ & $2.094(3)$ \\
\hline $\mathrm{Mo} 1-\mathrm{O} 2^{\mathrm{i}}$ & $1.894(3)$ & $\mathrm{Zn} 1-\mathrm{O} 1$ & $2.094(3)$ \\
\hline $\mathrm{Mo} 1-\mathrm{O} 2^{\mathrm{ii}}$ & $2.171(3)$ & $\mathrm{Zn} 1-\mathrm{O} 1^{\mathrm{vii}}$ & $2.274(4)$ \\
\hline $\mathrm{Mo} 1-\mathrm{O} 2^{\mathrm{iii}}$ & $2.171(3)$ & $\mathrm{Zn} 1-\mathrm{O} 1^{\text {viii }}$ & $2.274(4)$ \\
\hline $\mathrm{O} 1{ }^{\mathrm{i}}-\mathrm{Mo1}-\mathrm{O} 1$ & $104.7(2)$ & $\mathrm{O} 2^{\mathrm{iv}}-\mathrm{Zn} 1-\mathrm{O} 1$ & $95.54(14)$ \\
\hline
\end{tabular}




\begin{tabular}{|c|c|c|c|}
\hline $\mathrm{O} 1^{\mathrm{i}}-\mathrm{Mo} 1-\mathrm{O} 2$ & $97.25(15)$ & $\mathrm{O} 2^{\mathrm{v}}-\mathrm{Zn} 1-\mathrm{O} 1$ & $96.96(14)$ \\
\hline $\mathrm{O} 1-\mathrm{Mo1}-\mathrm{O} 2$ & $100.46(15)$ & 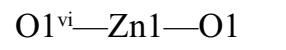 & $158.4(2)$ \\
\hline $\mathrm{O} 1^{\mathrm{i}}-\mathrm{Mo} 1-\mathrm{O} 2^{\mathrm{i}}$ & $100.46(15)$ & $\mathrm{O} 2^{\mathrm{iv}}-\mathrm{Zn} 1-\mathrm{O} 1^{\mathrm{vii}}$ & $162.81(14)$ \\
\hline $\mathrm{O} 1-\mathrm{Mo} 1-\mathrm{O} 2^{\mathrm{i}}$ & $97.25(15)$ & $\mathrm{O} 2^{\mathrm{v}}-\mathrm{Zn} 1-\mathrm{O} 1^{\mathrm{vii}}$ & $88.37(13)$ \\
\hline $\mathrm{O} 2-\mathrm{Mo} 1-\mathrm{O} 2^{\mathrm{i}}$ & $150.8(2)$ & 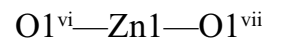 & $82.25(14)$ \\
\hline $\mathrm{O} 1^{\mathrm{i}}-\mathrm{Mo} 1-\mathrm{O} 2^{\mathrm{ii}}$ & $164.89(14)$ & $\mathrm{O} 1-\mathrm{Zn} 1-\mathrm{O} 1^{\mathrm{vii}}$ & $80.63(11)$ \\
\hline $\mathrm{O} 1-\mathrm{Mo} 1-\mathrm{O}^{\mathrm{ii}}$ & $88.90(14)$ & $\mathrm{O} 2^{\mathrm{iv}}-\mathrm{Zn} 1-\mathrm{O} 1^{\mathrm{viii}}$ & $88.37(13)$ \\
\hline $\mathrm{O} 2-\mathrm{Mo} 1-\mathrm{O} 2^{\mathrm{ii}}$ & $73.39(16)$ & $\mathrm{O} 2^{\mathrm{v}}-\mathrm{Zn} 1-\mathrm{O} 1^{\mathrm{vii}}$ & $162.81(14)$ \\
\hline $\mathrm{O} 2^{\mathrm{i}}-\mathrm{Mo} 1-\mathrm{O} 2^{\mathrm{ii}}$ & $84.01(11)$ & 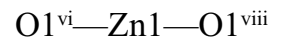 & $80.63(11)$ \\
\hline $\mathrm{O} 1^{\mathrm{i}}-\mathrm{Mo} 1-\mathrm{O} 2^{\mathrm{iii}}$ & $88.90(14)$ & $\mathrm{O} 1-\mathrm{Zn} 1-\mathrm{O}^{\mathrm{viii}}$ & $82.24(14)$ \\
\hline $\mathrm{O} 1-\mathrm{Mo} 1-\mathrm{O} 2^{\mathrm{iii}}$ & $164.89(15)$ & $\mathrm{O} 1^{\mathrm{vii}}-\mathrm{Zn} 1-\mathrm{O} 1^{\mathrm{viii}}$ & $74.53(18)$ \\
\hline $\mathrm{O} 2-\mathrm{Mo} 1-\mathrm{O} 2^{\mathrm{iii}}$ & $84.01(11)$ & $\mathrm{Mo} 1-\mathrm{O} 1-\mathrm{Zn} 1$ & $126.54(19)$ \\
\hline $\mathrm{O} 2^{\mathrm{i}}-\mathrm{Mo} 1-\mathrm{O} 2^{\mathrm{iii}}$ & $73.39(16)$ & Mo1-O1-Zn1 $1^{\text {viii }}$ & $133.83(18)$ \\
\hline $\mathrm{O} 2^{\mathrm{ii}}-\mathrm{Mo} 1-\mathrm{O} 2^{\mathrm{iii}}$ & $78.49(18)$ & $\mathrm{Zn} 1-\mathrm{O} 1-\mathrm{Zn} 1^{\mathrm{viii}}$ & $97.75(14)$ \\
\hline $\mathrm{O} 2^{\mathrm{iv}}-\mathrm{Zn} 1-\mathrm{O} 2^{\mathrm{v}}$ & $108.8(2)$ & Mo1-O2-Zn $1^{\text {ix }}$ & $125.93(18)$ \\
\hline $\mathrm{O} 2^{\mathrm{iv}}-\mathrm{Zn} 1-\mathrm{O} 1^{\mathrm{vi}}$ & $96.96(14)$ & $\mathrm{Mo} 1-\mathrm{O} 2-\mathrm{Mol}^{\mathrm{ii}}$ & $106.61(16)$ \\
\hline $\mathrm{O} 2^{\mathrm{v}}-\mathrm{Zn} 1-\mathrm{O}^{\mathrm{vi}}$ & $95.54(14)$ & $\mathrm{Zn} 1^{\mathrm{ix}}-\mathrm{O} 2-\mathrm{Mo}^{\mathrm{ii}}$ & $124.32(17)$ \\
\hline
\end{tabular}

Symmetry codes: (i) $-x+2, y,-z+1 / 2$; (ii) $-x+2,-y+2,-z+1$; (iii) $x,-y+2, z-1 / 2$; (iv) $x+1, y, z$; (v) $-x+2, y,-z+3 / 2$; (vi) $-x+3, y,-z+3 / 2$; (vii) $x,-y+1$, $z+1 / 2$; (viii) $-x+3,-y+1,-z+1$; (ix) $x-1, y, z$. 